UNIVERSITAS, Volumen 4, Número 1, 2013, 41-52, ISSN 2071-2574

(c) UNAN-León, Editorial Universitaria

\title{
DIFERENCIAS EN LA VIVENCIA DE SUCESOS VITALES ESTRESANTES EN NICARAGUA, CHILE Y ESPAÑA.
}

\section{Claudia Medina Sandino ${ }^{1}$, Alberto Berríos Ballesteros ${ }^{1}$, Sonia Panadero Herrero², Paulina Rincón González ${ }^{3}$, José Juan Vázquez Cabrera ${ }^{4 *}$}

1Carrera de Psicología. Facultad de Ciencias Médicas. Universidad Nacional Autónoma de Nicaragua (UNAN- León). Nicaragua.

2 Departamento de Personalidad, Evaluación y Tratamientos Psicológicos. Facultad de Psicología. Universidad Complutense de Madrid (UCM). España.

3 Facultad de Psicología. Universidad de Concepción. (UdeC). Chile.

2 Área de Psicología Social. Universidad de Alcalá (UAH). España.

\section{RESUMEN}

El artículo describe los resultados de un trabajo realizado con 481 estudiantes de psicología de cinco universidades de Nicaragua, Chile y España, tres países que presenten diferentes niveles de desarrollo socioeconómico. Se analiza el riesgo de padecer determinados sucesos vitales estresantes y la cantidad y características de los estresores padecidos, así como su relación con el nivel de desarrollo del país en que habitan los participantes, su clase social y el nivel de desesperanza manifestado por estos. La información se recogió utilizando un cuestionario autoaplicado. Los resultados obtenidos indican una mayor incidencia en la vivencia de sucesos vitales estresantes entre los participantes que habitan en los países con menores niveles de desarrollo, y entre quienes pertenecen a las clases sociales más desfavorecidas. Los resultados apuntan igualmente a una relación entre la cantidad de sucesos vitales padecidos y el nivel de desesperanza manifestado por los entrevistados.

Palabras clave: sucesos vitales estresantes, clases social, desesperanza, desarrollo.

\section{INTRODUCCIÓN.}

Diferentes trabajos de investigación han encontrado que el padecimiento de sucesos vitales estresantes se encuentra relacionado con el incremento del malestar psicológico (Lesniak, Rudman, Rector y Elkin, 2006), síntomas físicos (Cropley y Steptoe, 2005), pobre salud física y mental (Adams, Boscarino y Galea, 2006), trastornos mentales (Hackett, Hackett, Bhakta y Gowers, 2000) y problemas psicológicos y consecuencias negativas en la salud física y mental a corto y largo plazo (Brewin, Andrews y Valentine, 2000).

Por otro lado, la relación entre adversidad social y número de sucesos vitales estresantes padecidos se encuentra bien documentada, observándose que el padecimiento de un mayor número de sucesos vitales estresantes se encuentra asociado a la baja clase social y a situaciones de pobreza (Hackett, Hackett, Bhakta y Gowers, 2000; Lantz, House, Mero y Williams, 2005). Asimismo, distintas investigaciones han mostrado un incremento en la frecuencia de sucesos vitales estresantes entre las personas con mayores niveles de pobreza (Muñoz, Vázquez, Bermejo y Vázquez, 1999; Roll, Toro y Ortola, 1999).

Los bajos niveles de desarrollo socioeconómico de un país pueden incidir en una mayor exposición de su población a situaciones vitales estresantes por la existencia, entre otros aspectos, de unos deficientes o inexistentes servicios públicos y sistemas de protección

* $\quad$ Autor para correspondencia: Dr. José Juan Vázquez. Área de Psicología Social. Universidad de Alcalá. Aulario María de Guzmán. C/ San Cirilo, s/n. 28801. Alcalá de Henares (Madrid). España. Teléfono: 3491885 2400. Correo electrónico:jj.vazquez@uah.es. 
social, junto a mayores tasas de pobreza y elevados porcentajes de personas enmarcadas en las clases sociales más desfavorecidas. De hecho, la población de los países en desarrollo presentan niveles de salud inferiores (Krug, 2004).

Asimismo, los sucesos vitales estresantes, incluyendo aquellos de carácter menor, y sucesos traumáticos (Dixon, Rumford, Heppner y Lips, 1992), han aparecido fuertemente asociados con la desesperanza (Dixon, Heppner y Anderson, 1991; Haatainen, Tanskanen, Kylmä, Antikainen, Hintikka, Honkalampi, Koivumaa-Honkanen y Viinamäki, 2003). Además, si las personas con desesperanza experimentan más sucesos adversos, corren el riesgo de incrementar la duración de dicha desesperanza (Haatainen et al., 2003), dando lugar a un círculo vicioso de funestas consecuencias. La desesperanza es, asimismo, un factor relevante en el padecimiento de trastornos afectivos y en el incremento de la tasa de conducta suicida (Beck, Brown, Berchick, Stewart y Steer, 1990; Sullivan, 2003). De hecho, por cada 100.000 jóvenes de entre 15 y 24 años de edad, las tasas de suicidio se sitúan en 7,6 en Nicaragua; 6,3 en Chile y 4,7 en España (WHO, 2005). Aparentemente existe una correlación inversa entre tasa de suicidio y nivel desarrollo humano del país en que habitan los jóvenes.

En el presente trabajo se analizan los sucesos vitales estresantes padecidos por estudiantes de psicología (grupo homogéneo en edad y nivel cultural) de tres países con diferentes niveles de desarrollo, Nicaragua, Chile y España, que ocupan respectivamente los puestos 110, $40 \mathrm{y}$ 13 en el Índice de Desarrollo Humano (UNDP, 2008).

\section{DISEÑO METODOLÓGICO.}

\section{Muestra.}

En el presente estudio participaron 481 estudiantes de psicología. Concretamente participaron 209 estudiantes nicaragüenses, de la Universidad Nacional Autónoma de Nicaragua en León (167 estudiantes) y de la Universidad Autónoma de Chinandega (42 estudiantes); 156 estudiantes chilenos, de la Universidad de Concepción (81 estudiantes) y de la Universidad de Santo Tomás en Los Ángeles (75 estudiantes); y 116 estudiantes españoles, de la Universidad Complutense de Madrid.

La media de edad de los participantes (80,4\% mujeres y $19,6 \%$ varones) fue 21,87 años (d.t .=4,202).

\section{Instrumento.}

El diseño del cuestionario, de carácter autoaplicado, se realizó en España, donde fue pilotado en una muestra de estudiantes españoles de psicología que cursaban sus estudios en la Universidad Nacional de Educación a Distancia (UNED). En América, con la colaboración de profesores de las universidades participantes, se realizó una revisión del cuestionario para adecuarlo a las distintas variantes del español utilizadas en los diferentes países.

El cuestionario se dividía en diferentes secciones. En este artículo se presenta información sobre tres de ellas, concretamente:

- Datos sociodemográficos: se recogió información relativa al sexo, edad, estado civil, clase social de pertenencia y situación económica familiar.

- Desesperanza: se empleó la "Escala de Desesperanza" (Beck, Weissman, Lester y Trexler, 1974), instrumento utilizado 
internacionalmente que permite evaluar la actitud del entrevistado hacia las expectativas futuras y presenta unos adecuados criterios metodológicos (Bouvard, Charles, Guerin, Aimard y Cottraux, 1992; Steed, 2001).

- Sucesos Vitales Estresantes: se utilizó una adaptación de 20 ítems realizada a partir de un cuestionario que ha mostrado su eficacia en trabajos realizados con diferentes poblaciones de habla hispana (Muñoz, Panadero, Santos y Quiroga, 2005; Muñoz, Vázquez y Vázquez, 2003).

\section{Procedimiento.}

La aplicación del cuestionario fue colectiva. Con colaboración del profesorado y la dirección de los centros se accedió a los estudiantes en las aulas. Tras exponer el objeto de la investigación y el tratamiento que se daría a los datos, se solicitó el consentimiento informado, señalándose que en todo momento se respetaría el anonimato de quienes completasen el cuestionario.

Posteriormente se expuso la forma de completar el cuestionario y se explicó el significado de algunos ítems que, en el contexto americano, se observó que podían inducir a error. A continuación se solicitó a los estudiantes que respondiesen al cuestionario, sin establecer para ello un límite de tiempo. Durante la aplicación del cuestionario se encontraba presente en el aula un colaborador de la investigación para resolver las dudas que surgiesen.

\section{Análisis estadísticos.}

Para realizar el análisis de datos se utilizó el sistema de análisis estadístico y de gestión de datos SPSS (versión 15.0 para Windows). Para identificar las diferencias en la ocurrencia de los diferentes sucesos vitales entre las diferentes nacionalidades y clases sociales se utilizaron, para variables nominales el estadístico "Chi cuadrado" y para variables continuas pruebas $t$ de Student para muestras independientes y ANOVA de un factor. Además, para cada uno de los sucesos vitales estresantes considerados se realizó la estimación del riesgo para las personas de los diferentes países. Para ello se calculó las "odds ratio", con 95\% de intervalo de confianza (Rudas, 1998). Para calcular la correlación de la desesperanza con el número de sucesos vitales estresantes y con la clase social se empleó "Pearson" y "Tau-b" de Kendall respectivamente.

\section{RESULTADOS.}

Como se aprecia en la tabla 1, existen diferencias significativas entre los estudiantes de psicología nicaragüenses, chilenos y españoles en algunas variables sociodemográficas. Así, entre los nicaragüenses se observan los mayores porcentajes de estudiantes casados. Y, si bien la mayoría de entrevistados se autodefine de "clase media", a mayor desarrollo socioeconómico del país un mayor porcentaje de estudiantes se autodefinen de clase "media-alta" y un menor porcentaje de clase "media-baja". 
Tabla 1. Características sociodemográficas de los estudiantes de psicología nicaragüenses, chilenos y españoles.

\begin{tabular}{|c|c|c|c|c|c|c|}
\hline Características ( $\square 2 / F)$ (1) & $\begin{array}{c}\text { Nicaragua } \\
\quad(\mathrm{n}=208)\end{array}$ & $\begin{array}{c}\text { Chile } \\
(\mathrm{n}=156)\end{array}$ & $\begin{array}{l}\text { España } \\
(\mathrm{n}=116)\end{array}$ & $\begin{array}{c}\square 2 \\
\text { (Nicaragua- } \\
\text { Chile) }\end{array}$ & $\begin{array}{c}\square 2 \\
\text { (Nicaragua- } \\
\text { España) }\end{array}$ & $\begin{array}{c}\square 2 \\
\text { (Chile- } \\
\text { España) }\end{array}$ \\
\hline Edad (Media (dt)) & $\begin{array}{l}22,25 \\
(4,86)\end{array}$ & $\begin{array}{l}21,46 \\
(4,31)\end{array}$ & $\begin{array}{l}21,76 \\
(2,09)\end{array}$ & $-1,598$ & $-1,257$ & $-0,723$ \\
\hline Sexo & & & & $17,218 * * *$ & 0,089 & $10,163 * * *$ \\
\hline Varones & $13,5 \%$ & $31,4 \%$ & $14,7 \%$ & & & \\
\hline Mujeres & $86,5 \%$ & $68,6 \%$ & $85,3 \%$ & & & \\
\hline Estado Civil & & & & $14,114 * *$ & $16,966 * * *$ & 1,175 \\
\hline Solteros & $81,5 \%$ & $94,8 \%$ & $97,4 \%$ & & & \\
\hline Casados o viviendo en pareja & $17,6 \%$ & $5,2 \%$ & $2,6 \%$ & & & \\
\hline Separado o divorciado & $0,5 \%$ & $0 \%$ & $0 \%$ & & & \\
\hline Clase social & & & & $39,323 * * *$ & $81,956 * * *$ & $22,911 * * *$ \\
\hline Baja & $7,8 \%$ & $1,9 \%$ & $1,7 \%$ & & & \\
\hline Media baja & $38,5 \%$ & $16,0 \%$ & $5,2 \%$ & & & \\
\hline Media & $48,8 \%$ & $70,5 \%$ & $60,3 \%$ & & & \\
\hline Media alta & $3,4 \%$ & $11,5 \%$ & $31,9 \%$ & & & \\
\hline Alta & $1,5 \%$ & $0 \%$ & $0,9 \%$ & & & \\
\hline
\end{tabular}

$* \mathrm{p} \leq 0,05 ; * * \mathrm{p} \leq 0,01 ; * * * \mathrm{p} \leq 0,001$

Conforme desciende el nivel de desarrollo de sus respectivos países, los estudiantes (que no se diferencian significativamente en edad) manifiestan haber padecido un número significativamente mayor de sucesos vitales estresantes: de media, los "nicaragüenses" han padecido 3,53 sucesos de este tipo (d.t. $=2,243)$, los "chilenos" 2,27 (d.t.=1,777) y los “españoles” 1,50 (d.t. =1,720), observándose diferencias estadísticamente significativas entre "chilenos" y "nicaragüenses" $(\mathrm{t}=-5,507$; $\mathrm{p}=0,000)$, "españoles" y "nicaragüenses" $(\mathrm{t}=-8,220 ; \mathrm{p}=0,000) \quad \mathrm{y}$ "chilenos" $\mathrm{y}$ “españoles" ( $t=3,530 ; p=0,000)$.

Con respecto al tipo de acontecimientos estresantes padecidos, se observan diferencias significativas entre los estudiantes de los diferentes países en la mayor parte de sucesos vitales estresantes recogidos, tal como se observa en las tablas 2, 3 y 4 . 
Tabla 2: Comparación entre participantes españoles y chilenos en la frecuencia de sucesos vitales estresantes padecidos.

\begin{tabular}{|c|c|c|c|c|c|}
\hline Sucesos Vitales Estresantes & España & Chile & $\square 2$ & $\begin{array}{l}\text { Odds } \\
\text { ratio }\end{array}$ & $95 \%$ CI \\
\hline $\begin{array}{l}\text { Problemas de salud física o mental grave de alguno de los } \\
\text { padres antes de los } 18 \text { años }\end{array}$ & $24,6 \%$ & $23,1 \%$ & 0,051 & & \\
\hline $\begin{array}{l}\text { Problemas con el alcohol o drogas de alguno de los padres } \\
\text { antes de los } 18 \text { años }\end{array}$ & $10,3 \%$ & $13,5 \%$ & 0,668 & & \\
\hline Divorcio o separación de los padres antes de los 18 años & $11,2 \%$ & $17,3 \%$ & 2,035 & & \\
\hline Fuga del sitio en que vivía antes de los 18 años & $3,4 \%$ & $9,0 \%$ & 3,386 & & \\
\hline $\begin{array}{l}\text { Criado por personas diferentes de los padres (familiares, } \\
\text { familia adoptiva, orfelinato, etc.) antes de los } 18 \text { años }\end{array}$ & $1,7 \%$ & $12,2 \%$ & $10,208 * * *$ & 7,905 & $1,803-34,659$ \\
\hline Maltrato físico o sexual antes de los 18 años & $4,3 \%$ & $3,8 \%$ & 0,025 & & \\
\hline Muerte del padre & $4,3 \%$ & $5,1 \%$ & 0,105 & & \\
\hline Muerte de la madre & $1,7 \%$ & $4,5 \%$ & 1,588 & & \\
\hline Muerte del cónyuge/pareja & $0,0 \%$ & $1,9 \%$ & 2,256 & & \\
\hline Separación o divorcio & $0,0 \%$ & $0,6 \%$ & 0,751 & & \\
\hline Problemas económicos importantes & $18,1 \%$ & $34,0 \%$ & $8,462 * *$ & 2,328 & $1,307-4,146$ \\
\hline $\begin{array}{l}\text { Desarrollo de trabajos o actividades que le hayan separado } \\
\text { de su domicilio }\end{array}$ & $28,4 \%$ & $37,2 \%$ & 2,394 & & \\
\hline Problemas de desempleo importantes & $7,8 \%$ & $4,5 \%$ & 1,256 & & \\
\hline Pérdida de vivienda por desahucio, derribo u otras causas & $0,0 \%$ & $1,3 \%$ & 1,498 & & \\
\hline Enfermedades, lesiones o accidentes graves & $11,2 \%$ & $22,4 \%$ & $5,772 *$ & 2,292 & $1,151-4,563$ \\
\hline Problemas de salud mental grave & $6,9 \%$ & $2,6 \%$ & 2,961 & & \\
\hline Consumo excesivo de alcohol o drogas & $13,8 \%$ & $26,3 \%$ & $6,401 *$ & 2,248 & $1,189-4,251$ \\
\hline Malos tratos por parte de su pareja/cónyuge & $0,0 \%$ & $5,1 \%$ & $6,129^{*}$ & - & - \\
\hline Agresiones físicas o sexuales (después de los 18 años) & $3,4 \%$ & $9,0 \%$ & 0,603 & & \\
\hline
\end{tabular}

$* \mathrm{p} \leq 0,05 ; * * \mathrm{p} \leq 0,01 ; * * * \mathrm{p} \leq 0,001$ 
Como se observa en la Tabla 2, la ocurrencia de los sucesos vitales considerados en los estudiantes españoles y chilenos es muy similar (ver Tabla 1). Tan sólo aparecen diferencias estadísticamente significativas en el ítem Ser criado por personas diferentes de los padres, que ocurre con mayor frecuencia entre los estudiantes chilenos: más de uno de cada diez participantes chilenos no fue criado por sus padres (es decir, fue criado por otros familiares, por familia adoptiva, etc.) frente a casi dos de cada cien participantes españoles. Como indican las "odds ratio", el riesgo de ser criado por personas diferentes de los padres (es decir, por otros familiares, por familia adoptiva, etc.) durante la infancia es mucho mayor entre los estudiantes chilenos $(\mathrm{OR}=7,905,95 \% \mathrm{CI}=1,803-34,659)$.
También los participantes chilenos manifestaron haber tenido en mayor medida que los españoles problemas económicos importantes (34\% frente al 18\%), enfermedades, lesiones o accidentes graves (22,4 frente a la mitad entre los participantes españoles) y consumo excesivo de drogas o alcohol (26,3\% frente al 13,8\%).

Tabla 3: Comparación entre participantes españoles y nicaragüenses en la frecuencia de sucesos vitales estresantes padecidos.

\begin{tabular}{|c|c|c|c|c|c|}
\hline Sucesos Vitales Estresantes & España & $\begin{array}{l}\text { Nica- } \\
\text { ragua }\end{array}$ & $\square 2$ & $\begin{array}{l}\text { Odds } \\
\text { ratio }\end{array}$ & $95 \% \mathrm{CI}$ \\
\hline $\begin{array}{l}\text { Problemas de salud física o mental grave de alguno de los } \\
\text { padres antes de los } 18 \text { años }\end{array}$ & $24,6 \%$ & $28,4 \%$ & 0,555 & & \\
\hline $\begin{array}{l}\text { Problemas con el alcohol o drogas de alguno de los } \\
\text { padres antes de los } 18 \text { años }\end{array}$ & $10,3 \%$ & $34,8 \%$ & $23,072 * * *$ & 4,622 & $2,38-8,96$ \\
\hline Divorcio o separación de los padres antes de los 18 años & $11,2 \%$ & $47,1 \%$ & $42,321 * * *$ & 7,043 & $3,717-13,344$ \\
\hline Fuga del sitio en que vivía antes de los 18 años & $3,4 \%$ & $5,8 \%$ & 0,871 & & \\
\hline $\begin{array}{l}\text { Criado por personas diferentes de los padres (familiares, } \\
\text { familia adoptiva, orfelinato, etc.) antes de los } 18 \text { años }\end{array}$ & $1,7 \%$ & $14,6 \%$ & $13,668 * * *$ & 9,716 & $2,278-41,446$ \\
\hline Maltrato físico o sexual antes de los 18 años & $4,3 \%$ & $19,3 \%$ & $13,974 * * *$ & 5,317 & $2,036-13,89$ \\
\hline Muerte del padre & $4,3 \%$ & $13,1 \%$ & $6,416^{*}$ & 3,349 & $1,253-8,951$ \\
\hline Muerte de la madre & $1,7 \%$ & $2,4 \%$ & 0,177 & & \\
\hline Muerte del cónyuge/pareja & $0,0 \%$ & $1,5 \%$ & 1,793 & & \\
\hline Separación o divorcio & $0,0 \%$ & $4,3 \%$ & $5,125^{*}$ & - & - \\
\hline
\end{tabular}




\begin{tabular}{|c|c|c|c|c|c|}
\hline Problemas económicos importantes & $18,1 \%$ & $71,5 \%$ & $84,959 * * *$ & 11,348 & $6,477-19,881$ \\
\hline $\begin{array}{l}\text { Desarrollo de trabajos o actividades que le hayan } \\
\text { separado de su domicilio }\end{array}$ & $28,4 \%$ & $30,4 \%$ & 0,134 & & \\
\hline Problemas de desempleo importantes & $7,8 \%$ & $25,1 \%$ & $14,520 * * *$ & 3,990 & $1,881-8,462$ \\
\hline Pérdida de vivienda por desahucio, derribo u otras causas & $0,0 \%$ & $4,9 \%$ & $5,840^{*}$ & - & - \\
\hline Enfermedades, lesiones o accidentes graves & $11,2 \%$ & $31,4 \%$ & $16,551 * * *$ & 3,627 & $1,899-6,928$ \\
\hline Problemas de salud mental grave & $6,9 \%$ & $1,9 \%$ & $5,078^{*}$ & 0,267 & $0,079-0,908$ \\
\hline Consumo excesivo de alcohol o drogas & $13,8 \%$ & $7,8 \%$ & 2,909 & & \\
\hline Malos tratos por parte de su pareja/cónyuge & $0,0 \%$ & $10,3 \%$ & $12,784 * * *$ & - & - \\
\hline Agresiones físicas o sexuales (después de los 18 años) & $3,4 \%$ & $7,4 \%$ & 2,019 & & \\
\hline
\end{tabular}

$* \mathrm{p} \leq 0,05 ; * * \mathrm{p} \leq 0,01 ; * * * \mathrm{p} \leq 0,001$

Como puede verse en la Tabla 3, entre los participantes españoles y nicaragüenses existen diferencias estadísticamente significativas en el padecimiento de un elevado número de sucesos vitales estresantes. Entre los sucesos vitales vividos antes de los 18 años de edad aparecen algunas diferencias, que en todos los casos muestran una peor situación entre los estudiantes nicaragüenses. Las "odds ratio" indican un riesgo mucho mayor entre los nicaragüenses de padecer problemas relacionados con el consumo de drogas $o$ alcohol en los padres ( $\mathrm{OR}=4,622 ; 95 \%$ $\mathrm{CI}=2,38-8,96)$, divorcio o separación de los padres $(\mathrm{OR}=7,043 ; 95 \% \mathrm{CI}=3,717-13,344)$, ser criado por personas diferentes de los padres $(\mathrm{OR}=9,716 ; 95 \% \mathrm{CI}=2,278-41,446) \mathrm{y}$ maltrato físico o sexual antes de los 18 años $(\mathrm{OR}=5,317 ; 95 \% \mathrm{CI}=2,036-13,89)$.

También se observan diferencias importantes en aspectos económicos, que igualmente apuntan a una peor situación de los estudiantes nicaragüenses: casi tres de cada cuatro nicaragüenses han tenido problemas económicos importantes (frente a un 18\% de los españoles) y uno de cada cuatro tuvieron problemas de desempleo importantes (frente a un $7,8 \%$ de los españoles). Las "odds ratio" indican que el riesgo de padecer problemas económicos y especialmente problemas de desempleo importantes es mucho mayor entre los participantes nicaragüenses $(\mathrm{OR}=11,348$; $\mathrm{CI}=6,477-19,881$ y $\mathrm{OR}=3,990 ; 95 \% \mathrm{CI}=1,881$ 8,462 respectivamente).

Tan sólo uno de los sucesos considerados aparece de forma significativamente más frecuente entre los estudiantes españoles: el riesgo de padecer problemas de salud mental grave es mayor entre los estudiantes españoles $(\mathrm{OR}=0,267$; CI 95\%=0,079-0,908). Sin embargo, en cuanto a la salud física, los datos apuntan a una peor situación de los estudiantes nicaragüenses: más de un 30\% de ellos informan de enfermedades, lesiones o accidentes graves frente a un $11,2 \%$ de los españoles. El riesgo este tipo de problemas es mayor de nuevo entre los nicaragüenses $(\mathrm{OR}=3,627$; CI 95\%=1,899-6,928). 
Tabla 4: Comparación entre participantes chilenos y nicaragüenses en la frecuencia de sucesos vitales estresantes padecidos.

\begin{tabular}{|c|c|c|c|c|c|}
\hline Sucesos Vitales Estresantes & Chile & $\begin{array}{l}\mathrm{N} \text { i c a - } \\
\text { ragua }\end{array}$ & $\square 2$ & $\begin{array}{l}\text { Od d s } \\
\text { ratio }\end{array}$ & $95 \% \mathrm{CI}$ \\
\hline $\begin{array}{l}\text { Problemas de salud física o mental grave de alguno de los } \\
\text { padres antes de los } 18 \text { años }\end{array}$ & $23,1 \%$ & $28,4 \%$ & 1,158 & - & - \\
\hline $\begin{array}{l}\text { Problemas con el alcohol o drogas de alguno de los padres } \\
\text { antes de los } 18 \text { años }\end{array}$ & $13,5 \%$ & $34,8 \%$ & $20,647 * * *$ & 3,378 & $1,965-5,807$ \\
\hline Divorcio o separación de los padres antes de los 18 años & $17,3 \%$ & $47,1 \%$ & $34,354 * * *$ & 4,214 & $2,561-6,933$ \\
\hline Fuga del sitio en que vivía antes de los 18 años & $9,0 \%$ & $5,8 \%$ & 1,433 & - & - \\
\hline $\begin{array}{l}\text { Criado por personas diferentes de los padres (familiares, } \\
\text { familia adoptiva, orfelinato, etc.) antes de los } 18 \text { años }\end{array}$ & $12,2 \%$ & $14,6 \%$ & 0,431 & - & - \\
\hline Maltrato físico o sexual antes de los 18 años & $3,8 \%$ & $19,3 \%$ & $18,726^{* * *}$ & 5,868 & $2,419-14,236$ \\
\hline Muerte del padre & $5,1 \%$ & $13,1 \%$ & $6,378^{*}$ & 2,772 & $1,222-6,284$ \\
\hline Muerte de la madre & $4,5 \%$ & $2,4 \%$ & 1,156 & - & - \\
\hline Muerte del cónyuge/pareja & $1,9 \%$ & $1,5 \%$ & 0,080 & - & - \\
\hline Separación o divorcio & $0,6 \%$ & $4,3 \%$ & $4,398^{*}$ & 6,921 & $0,856-55,959$ \\
\hline Problemas económicos importantes & $34,0 \%$ & $71,5 \%$ & $50,686^{* * *}$ & 4,875 & $3,114-7,631$ \\
\hline $\begin{array}{l}\text { Desarrollo de trabajos o actividades que le hayan separado } \\
\text { de su domicilio }\end{array}$ & $37,2 \%$ & $30,4 \%$ & 1,955 & & \\
\hline Problemas de desempleo importantes & $4,5 \%$ & $25,1 \%$ & $27,396 * * *$ & 7,095 & $3,115-16,158$ \\
\hline Pérdida de vivienda por desahucio, derribo u otras causas & $1,3 \%$ & $4,9 \%$ & 3,565 & & \\
\hline Enfermedades, lesiones o accidentes graves & $22,4 \%$ & $31,4 \%$ & $3,582 *$ & 1,582 & $0,982-2,550$ \\
\hline Problemas de salud mental grave & $2,6 \%$ & $1,9 \%$ & 0,159 & & \\
\hline Consumo excesivo de alcohol o drogas & $26,3 \%$ & $7,8 \%$ & $22,835 * * *$ & 0,237 & $0,127-0,441$ \\
\hline Malos tratos por parte de su pareja/cónyuge & $5,1 \%$ & $10,3 \%$ & 3,154 & & \\
\hline Agresiones físicas o sexuales (después de los 18 años) & $9,0 \%$ & $7,4 \%$ & $5,428^{*}$ & 4,021 & $1,143-14,146$ \\
\hline
\end{tabular}

$* \mathrm{p} \leq 0,05 ; * * \mathrm{p} \leq 0,01 ; * * * \mathrm{p} \leq 0,001$ 
Como se recoge en la Tabla 4, existen diferencias estadísticamente significativas entre los estudiantes nicaragüenses y chilenos participantes en este trabajo en bastantes de los sucesos considerados, relacionados con los aspectos más diversos (salud, económicos, etc.) y que señalan una peor situación entre los estudiantes nicaragüenses. Sobre los sucesos vitales estresantes padecidos por los entrevistados antes de los 18 años de edad, entre los estudiantes nicaragüenses se observa un riesgo mucho mayor de haber tenido padres con problemas de alcohol o drogas $(\mathrm{OR}=3,378 ; 95 \% \mathrm{CI}=1,965-5,807)$, divorciados $(\mathrm{OR}=4,214 ; 95 \% \mathrm{CI}=2,561-6,933)$ o de haber sufrido maltrato físico o sexual antes de los 18 años de edad $(\mathrm{OR}=5,868 ; 95 \%$ $\mathrm{CI}=2,419-14,236)$. Asimismo, se observan diferencias estadísticamente significativas en agresiones físicas y sexuales después de los 18 años de edad, de forma que el riesgo entre los estudiantes nicaragüenses de sufrir agresiones físicas o sexuales parece importante, conforme se desprende de las "odds ratio" recogidos en la Tabla $4(\mathrm{OR}=4,021 ; 95 \% \mathrm{CI}=1,143-14,146)$.

También entre estudiantes nicaragüenses y chilenos aparecen diferencias en el padecimiento de sucesos vitales estresantes relacionados con aspectos económicos, como resulta esperable por el diferente nivel de desarrollo de ambos países. Casi uno de cada cuatro nicaragüenses manifiesta haber sufrido problemas de desempleo importantes (frente a un $4,5 \%$ de los chilenos) y más de un $70 \%$ problemas económicos importantes (el doble de lo encontrado entre los chilenos). De nuevo las "odds ratio" muestran una importante relación $\quad(\mathrm{OR}=7,095 ; \quad 95 \% \quad \mathrm{CI}=3,115$ 16,158 y $\mathrm{OR}=4,875 ; 95 \% \mathrm{CI}=3,114-7,631$ respectivamente).

Tan sólo en uno de los sucesos vitales estresantes recogidos aparece una peor situación entre los estudiantes chilenos que entre los nicaragüenses: el consumo excesivo de alcohol o drogas, que afecta a más de uno de cada cuatro chilenos (frente al 7,8\% de los nicaragüenses).

Además de las diferencias en función de la nacionalidad, de los datos obtenidos en el trabajo se desprende la existencia de diferencias significativas en el número de sucesos vitales estresantes padecidos en función de la clase social manifestada por los estudiantes de psicología. Así, conforme los entrevistados manifiestan pertenecer a una clase social más baja, se observa que han padecido una mayor cantidad de sucesos vitales estresantes: los estudiantes que manifiestan ser de "clase baja" han padecido 4,25 sucesos de media (d.t. $=2,543)$, los de "clase media baja" 3,62 sucesos (d.t.= 2,603), los de "clase media" 2,37 sucesos (d.t. $=2,024)$, los de "clase media alta" 1,93 sucesos (d.t.=1,652) y los de "clase alta" 1,75 sucesos (d.t.=1,258). En este sentido se observan diferencias estadísticamente significativas entre quienes manifiestan pertenecer a la "clase alta" y quienes pertenecen a las clases "media baja" $(\mathrm{t}=-2,754 ; \mathrm{p}=0,050)$ y "baja" $(\mathrm{t}=-2,795 ; \mathrm{p}=0,19)$; así como entre quienes pertenecen a la "clase media alta" y quienes pertenecen a las clases "baja" $(\mathrm{t}=-4,421 ; \mathrm{p}=$ $0,000)$ y "media baja" ( $t=-4,543 ; \mathrm{p}=0,000) ; \mathrm{y}$ quienes pertenecen a la "clase media" y los de las clases "media baja" $(\mathrm{t}=-4,832 ; \mathrm{p}=0,00) \mathrm{y}$ "baja" (t=-3,547; $\mathrm{p}=0,000)$.

Los datos de este trabajo también apuntan la relación entre el número de sucesos vitales parecidos y la puntuación en desesperanza. La correlación entre ambas variables es significativa, como puede verse en la Tabla 5, es decir, a mayor número de sucesos vitales estresantes mayor nivel de desesperanza. También aparece una correlación significativa entre la clase social y el nivel de desesperanza: según aumenta la clase social disminuye el nivel de desesperanza. 
Tabla 5. Correlación entre desesperanza y número de sucesos vitales estresantes y clase social

\begin{tabular}{l|l}
\hline & \multicolumn{1}{c}{ Desesperanza } \\
\hline No de Sucesos Vitales Estresantes & $0,104 *(1)$ \\
\hline Clase social & $-0,096^{*}(2)$ \\
\hline
\end{tabular}

(1) Pearson

(2) Tau_b de Kendall

\section{DISCUSIÓN.}

En línea de lo recogido en la literatura científica por autores como Lantz et al. (2005) o Hackett et al. (2000), los resultados del presente trabajo ahondan en la relación entre situaciones de desventaja socioeconómica y padecimiento de un mayor número de sucesos vitales estresantes, de forma que son los entrevistados que manifiestan pertenecer a las clases sociales menos favorecidas $y$ quienes habitan en los países con menor nivel de desarrollo los que manifiestan haber vivido una mayor cantidad de sucesos vitales estresantes a lo largo de su vida.

Ahora bien, dado que es en los estados con menores niveles de desarrollo donde se da una mayor presencia de entrevistados que manifiestan pertenecer a las clases sociales menos favorecidas, parece lógico que la vivencia de determinados eventos vitales estresantes como "padecer problemas económicos importantes" se incremente conforme los estados presentan menores tasas de desarrollo. Algo similar sucede con las diferencias de frecuencia en el padecimiento de "enfermedades, lesiones o accidentes graves", aspecto que parece encontrarse inversamente relacionada con el nivel de desarrollo presente en cada uno de los países. Este mayor padecimiento de "enfermedades, lesiones o accidentes graves" podría tener su explicación en cuestiones como el estilo de vida de los participantes de los diferentes países (condicionados por aspectos como la calidad de los alimentos disponibles, los niveles de protección laboral, los medios de comunicación, etc.) o las diferencias en las prestaciones sanitarias (número de médicos por habitante, posibilidades de adquisición de medicamentos, calidad del sistema hospitalario, etc.), cuestiones todas ellas relacionadas en gran medida con los niveles de desarrollo de cada uno de los países.

Sin embargo, las diferencias en la frecuencia de estresores vividos observada entre los diferentes estados no se circunscriben a los aspectos económicos y sanitarios, sino que abarcan otras circunstancias que parecen tener una relación indirecta con la situación económica del país. Por ejemplo, en los estados con menores tasas de desarrollo se observa que los entrevistados han padecido de forma más frecuente sucesos estresantes durante la infancia y adolescencia: problemas de consumo de alcohol o drogas por parte de los padres, maltrato físico o sexual, muerte del padre, haber sido criados por personas diferentes a sus padres o haber vivido la separación o divorcio de los padres. Este último aspecto es especialmente frecuente entre los estudiantes de Nicaragua, afectando 
prácticamente a la mitad de los mismos, mientras entre los estudiantes chilenos la frecuencia se reduce a uno de cada cinco, lo que no deja de resultar llamativo si se considera que la ley del divorcio en este último país es de aprobación muy reciente: 17 de mayo de 2004.

En los estados con menores niveles de desarrollo, los datos obtenidos indican que los matrimonios parecen tener lugar a edades más jóvenes, derivando posteriormente hacia mayores tasas de desestructuración familiar, abandono, malos tratos y violencia intrafamilar, observándose, por ejemplo, mayores tasas de maltrato por parte de las parejas: aproximadamente una de cada diez estudiantes mujeres nicaragüenses y una de cada veinte chilenas habían sufrido malos tratos por parte de su pareja a lo largo de su vida, mientras ninguna estudiante española informó haber padecido violencia por parte de su pareja. Sin embargo, aún constatándose importantes diferencias entre los tres países en cuanto a la vivencia de este tipo de acontecimientos, resulta difícil determinar si esto se encuentra vinculado a los diferentes niveles de pobreza, o bien a aspectos de carácter cultural propios de las sociedades donde se desarrolló el estudio.

También se observan en el estudio diferencias importantes en cuanto a la vivencia de otros sucesos estresantes como la pérdida de vivienda, de tal forma que estudiantes de Nicaragua manifiestan haber perdido en mayor medida la vivienda donde habitaban (aproximadamente un 5\%), aspecto que podría explicarse en cierta medida por los mayores niveles de precariedad económica, a lo que se sumaría la mayor incidencia de fenómenos naturales con consecuencias catastróficas en la zona: huracanes, tsunamis, inundaciones, erupciones volcánicas...
En cuanto a aspectos como el consumo excesivo de alcohol u otras drogas, estos haberse dado especialmente entre los estudiantes chilenos. En este sentido, aspectos culturales como la permisividad en el consumo de alcohol por parte de las mujeres (que supone el $80 \%$ de nuestra muestra) y la subjetividad asociada al concepto "consumo excesivo" pueden encontrarse detrás de los resultados obtenidos.

\section{AGRADECIMIENTOS.}

Agradecemos la colaboración en la realización del presenten trabajo de la Licenciada Victoria Sandino y a la Doctora María Crespo, así como a la autoridades de las universidades participantes $\mathrm{y}$, muy especialmente, a los alumnos de psicología de las distintas universidades que de forma desinteresada colaboraron en la investigación.

\section{REFERENCIAS BIBLIOGRÁFICAS.}

ADAMS, R.E., BOSCARINO, J.A. y GALEA, S. (2006). "Social and psychological resources and health outcomes after the World Trade Center disaster". Social Science and Medicine, 62 (1), 176-188.

BECK, A.T., WEISSMAN A., LESTER D. y TREXLER L. (1974). "The Measurement of Pessimism: the Hopelessness Scale". Journal of Consulting and Clinical Psychology, 42, 861-865.

BECK, A.T., BROWN, G., BERCHICK, R.J., STEWART, B.L. y STEER, R.A. (1990). "Relationship between hopelessness and ultimate suicide: A replication with psychiatric outpatients". American Journal of Psychiatry, 147, 190-195.

BOUVARD, M., CHARLES, S., GUERIN, J., AIMARD, G. y COTTRAUX, J. (1992). "Study of Beck's hopelessness scale. Validation and factor analysis". Encephale, 18, 237-40.

BREWIN, C.R. y ANDREWS, B. (2000) "Metaanalysis of risk factors for posttraumatic stress disorder in trauma-exposed adults". Journal of Consulting \& Clinical Psychology, 68, 748-757. 
BRUGHA, T.S. y CRAGG, D. (1990). "The List of Threatening Experiences: The reliability and validity of a brief life events questionnaire". Acta Psychiatrica Scandinavica, 82, 77-81.

CROPLEY, M. y STEPTOE, A. (2005). "Social support, life events and physical symptoms: A prospective study of chronic and recent life stress in men and women". Psychology, Health, \& Medicine; 10, 317-325.

DIXON, W.A., HEPPNER, P.P. y ANDERSON, W.P. (1991). "Problem-solving appraisal, stress, hopelessness, and suicide ideation in a college population". Journal of Counselling Psychology, 38, 51-56.

DIXON, W.A., RUMFORD, K.G., HEPPNER, P.P. y LIPS, B.J. (1992). "Use of different sources of stress to predict hopelessness and suicide in a college population". Journal of Counselling Psychology, 39, 342-349.

HAATAINEN, K.M., TANSKANEN, A., KYLMÄ, J., ANTIKAINEN, R., HINTIKKA, J., HONKALAMPI, K., KOIVUMAA-HONKANEN, H. y VIINAMÄKI, H. (2003). "Life events are important in the course of hopelessness: A 2-year follow-up study in a general population". Social Psychiatry \& Psychiatric Epidemiology; 38, 436-442.

HACKETT, R., HACKETT, L., BHAKTA, P. y GOWERS, S. (2000). "Life events in a south Indian population and their association with psychiatric disorder in children". International Journal of Social Psychiatry, 46, 201-207.

KRUG, E.G. (2004). "Injury surveillance is key to preventing injuries”. Lancet, 364 (9445), 1563-1566.

LANTZ, P.M., HOUSE, J.S., MERO, R.P. y WILLIAMS, D.R. (2005). "Stress, life events, and socioeconomic disparities in health: results from the Americans' Changing Lives Study". Journal of health and social behaviour $246,274-288$.
LESNIAK, K.T., RUDMAN, W., RECTOR, M.B. y ELKIN, T. (2006). "Psychological distress, stressful life events, and religiosity in younger African American adults". Mental Health, Religion \& Culture, 9 (1), 15-28.

MUÑOZ, M., VÁZQUEZ, C., BERMEJO, M. y VÁZQUEZ, J.J. (1999). "Stressful life events among homeless people: Quantity, types, timing, and perceived causality". Journal of Community Psychology, 27, 73-83.

UNDP (2008). Informe sobre desarrollo humano 2007-2008. La lucha contra el cambio climático: solidaridad frente a un mundo dividido. Nueva York: United Nations Development Program.

STEED, L. (2001). "Further Validity and Reliability Evidence for Beck Hopelessness Scale Scores in a Nonclinical Sample". Educational and Psychological Measurement, 61, 303-316.

ROLL, C.N., TORO, P.A. y ORTOLA, G.L. (1999). "Characteristics and experiences of homeless adults: A comparison of single men, single women, and women with children". Journal of Community Psychology, 27, 189-198.

SULLIVAN, M.D. (2003). "Hope and hopelessness at the end of life". American Journal of Geriatric Psychiatry, 11, 393-405.

VÁZQUEZ, J.J., PANADERO, S. y RINCÓN, P.P. (2006). "Acción política no convencional en universitarios españoles, chilenos, salvadoreños y nicaragüenses". Psicología Politica, 33, 25-41.

VÁZQUEZ, J.J., PANADERO, S. y RINCÓN, P. (2010). "Stressful life events and suicidal behaviour in countries with different development levels: Nicaragua, El Salvador, Chile and Spain". Journal of Community and Applied Social Psychology, 20, 288298

RUDAS, T. (1998). Odds ratios in the analysis of contingency tables. Londres: SAGE Publications. 